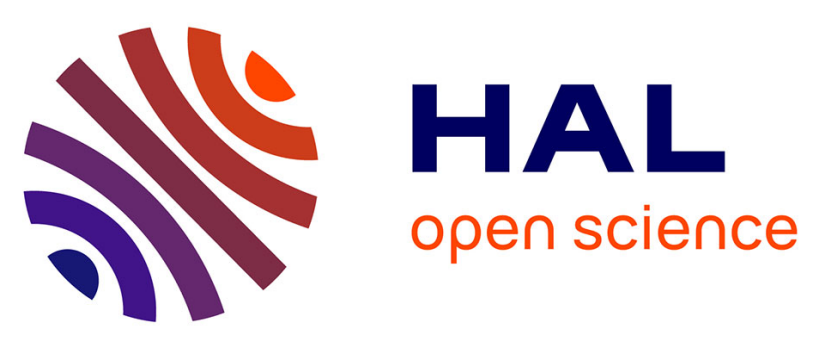

\title{
Structural Evidence That the Polymerization Rate Dictates Order and Intrinsic Strain Generation in Photocured Methacrylate Biomedical Polymers
}

Slobodan Sirovica, Maximilian W. A. Skoda, Maciej Podgorski, Paul B. J. Thompson, William M. Palin, Yilan Guo, Andrew J. Smith, Karun Dewan, Owen Addison, Richard A. Martin

\section{To cite this version:}

Slobodan Sirovica, Maximilian W. A. Skoda, Maciej Podgorski, Paul B. J. Thompson, William M. Palin, et al.. Structural Evidence That the Polymerization Rate Dictates Order and Intrinsic Strain Generation in Photocured Methacrylate Biomedical Polymers. Macromolecules, 2019, 52 (14), pp.5377-5388. 10.1021/acs.macromol.9b00133 . hal-02976351

\section{HAL Id: hal-02976351 \\ https://hal.science/hal-02976351}

Submitted on 23 Oct 2020

HAL is a multi-disciplinary open access archive for the deposit and dissemination of scientific research documents, whether they are published or not. The documents may come from teaching and research institutions in France or abroad, or from public or private research centers.
L'archive ouverte pluridisciplinaire HAL, est destinée au dépôt et à la diffusion de documents scientifiques de niveau recherche, publiés ou non, émanant des établissements d'enseignement et de recherche français ou étrangers, des laboratoires publics ou privés. 


\title{
Structural Evidence That the Polymerization Rate Dictates Order and Intrinsic Strain Generation in Photocured Methacrylate Biomedical Polymers
}

\author{
Slobodan Sirovica, ${ }^{\dagger}$ Maximilian W. A. Skoda, ${ }_{\nabla}^{\ddagger}$ Maciej Podgorski, ${ }^{\S}, \|$ Paul B. J. Thompson, ${ }^{\perp}$
} William M. Palin," Yilan Guo, ${ }^{\nabla}$ Andrew J. Smith, ${ }^{\mathbb{I}}$ Karun Dewan, $^{\#}$ Owen Addison, ${ }^{*, \#, \nabla, \triangle \odot}$ and Richard A. Martin*, ${ }^{*}, \triangle 0$

\footnotetext{
${ }^{\dagger}$ Aston Institute of Materials Research, School of Engineering \& Applied Science, University of Aston, Birmingham B4 7ET, U.K.

${ }^{\ddagger}$ ISIS Pulsed Neutron and Muon Source, Science and Technology Facilities Council, Rutherford Appleton Laboratory, Harwell Science and Innovation Campus, Didcot OX11 0QX, U.K.

${ }^{\S}$ Department of Chemical and Biological Engineering, University of Colorado Boulder, 596 UCB Boulder, Colorado 80309, United States

"Faculty of Chemistry, Department of Polymer Chemistry, Maria Curie-Sklodowska University, Gliniana Street 33, 20-614 Lublin, Poland

${ }^{\perp}$ XMaS The UK CRG, ESRF-The European Synchrotron, Grenoble 38000, France

${ }^{\#}$ College of Medical and Dental Sciences, Institute of Clinical Sciences, School of Dentistry, University of Birmingham, Mill Pool Way, Edgbaston, Birmingham B5 7EG, U.K.

${ }^{\nabla}$ Faculty of Medicine and Dentistry, University of Alberta, Edmonton T6G 1C9, Canada

${ }^{\mathbb{I}}$ Diamond Light Source Ltd., Diamond House, Harwell Science and Innovation Campus, Didcot, Oxfordshire OX11 0DE, U.K.

Supporting Information
}

\begin{abstract}
The influence of reaction rate on the evolving polymer structure of photo-activated dimethacrylate biomedical resins was investigated using neutron and in situ synchrotron X-ray scattering with simultaneous Fourier-transform-near-infrared spectroscopy. Previous studies have correlated the degree of reactive group conversion with mechanical properties, but the impact of polymerization rate on the resultant polymer structure is unknown. Here, we demonstrate that the medium-range structural order at the functional end groups of these

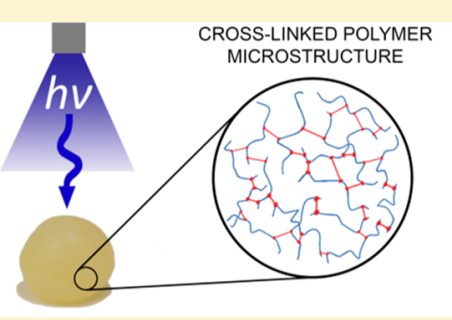

FINAL POLYMER CHAIN SEGMENT STRUCTURE materials is dependent on the reaction rate. Accelerating polymerization increases correlation lengths in the methacrylate end groups but reduces the medium-range structural order per converted vinyl bond when compared with more slowly polymerized systems. At faster rates of polymerization, the conformation of atoms at the reacting end group can become fixed into the polymer structure at the onset of autodeceleration, storing residual strain. Neutron scattering confirms that the structural differences observed are reproduced at longer length scales. This effect is not as prominent in systems polymerized at slower rates despite similar final degrees of reactive group conversion. Results suggest that current interpretations of these materials, which extrapolate mechanical properties from conversion, may be incomplete. Accelerating polymerization can introduce structural differences, which will dictate residual strain and may ultimately explain the discrepancies in the predictive modeling of the mechanical behavior of these materials using conventional techniques.
\end{abstract}

\section{INTRODUCTION}

Photo-initiated methacrylate polymers are of great biomedical importance as they provide, when compared with chemically cured alternatives, extended working times for the operator to place and manipulate the material and more rapid setting times once the correct material positioning has been achieved. Photo-initiated methacrylate polymer-based materials are routinely used in orthopedic surgery and, in particular, in dentistry where they are combined with inorganic filler particles to form resin-based composites. The physiochemical properties of these materials are highly sensitive to photo- polymerization variables which impact on the degree of reactive group conversion and the development of transient and residual stresses, which ultimately affect clinical performance. $^{1-3}$ The degree of reactive group conversion is typically measured using Fourier-transform infrared (FTIR) or Raman spectroscopy but fails to provide insight into the architecture of the cross-linked polymer structure and the development of

Received: January 18, 2019

Revised: May 15, 2019

Published: July 10, 2019 
<smiles>C=C(C)C(=O)OCCOCCOCCOC(=O)C(=C)C</smiles><smiles>CC(C)(C)OC(=O)C(=O)OCCOCCOCCOC(=O)C(=O)O</smiles>

Figure 1. (Top) TEGDMA. (Bottom) Partially deuterated TEGDMA monomer with $45.5 \%$ deuteration by molecular weight of the original hydrogen atoms.

strains. This work explores for the first time the effects of polymerization rate on the development of the medium-range ( $\sim 4-15 \AA)$ polymer structure of the most commonly encountered photo-initiated biomedical resin polymers based on methacrylate chemistry, which are used ubiquitously in contemporary dental care.

The majority of contemporary dental resin-composite filling materials are composed of a dimethacrylate polymer matrix that consist of a viscous monomer and reactive low molecular weight diluent monomer(s), combined with nanoscale and/or micron-scale inorganic filler particles. Following application to the tooth surface, the composite is "demand-set" by light excitation of a photo-initiator species dispersed within the resin matrix, initiating free radical polymerization and generating a solid three-dimensional cross-linked polymer network., These matrices typically achieve up to $\sim 80 \%$ conversion of the starting reagents to a polymer structure ${ }^{6,7}$ A higher degree of conversion (DC) has been shown to significantly influence resultant mechanical properties, conferring an increased flexural strength, surface hardness, ${ }^{8}$ elastic modulus, and wear resistance. ${ }^{9}$ It has, therefore, been proposed that the final mechanical properties of the composite are determined by the maximum $\mathrm{DC}\left(\mathrm{DC}_{\max }\right)$, which itself can be manipulated by altering the intensity of, and exposure to, the activating light source. ${ }^{10-12}$ It has been suggested that $\mathrm{DC}_{\max }$ is proportional to the total photon delivery irrespective of how they are delivered, a concept known as exposure reciprocity. ${ }^{13,14}$ However, theoretical and experimental approaches have shown that reactions that do not have a first-order dependency on the impinging light intensity, such as free radical photopolymerization and associated bimolecular processes, do not and should not obey the concept of reciprocity. ${ }^{15}$ Monomer structure, ${ }^{16,17}$ viscosity, co-monomer concentration, ${ }^{18-20}$ and differences in quantum efficiency ${ }^{21}$ and absorption spectra $^{22,23}$ of photo-initiator species will all impact on the mobility, polymerization rate, and $\mathrm{DC}_{\max }$ of these systems. Photo-activated resin composites, therefore, represent a complex system, and an understanding of polymerization cannot be obtained solely from the measurement of $\mathrm{DC}_{\max }$.

Several groups have instead varied the reaction rate and demonstrated a parabolic relationship between the polymerization rate, DC, and the final mechanical properties ${ }^{24}$ including tensile strength, toughness, hardness, and the magnitude of volumetric shrinkage of the polymer on polymerization. ${ }^{3,25-27}$ To date, these differences in mechanical properties, for similarly converted resin matrices polymerized at different rates, have been ascribed to the cross-link density and its effect on network architecture. Accelerating polymerization reduces the lifetime of radical species with respect to bimolecular termination, ${ }^{27}$ limiting the degree of cross-linkage and lowering the strength of the resultant matrix.

Accordingly, efforts have been made to optimize the resin matrix and photo-initiator system composition in terms of polymerization rate and $\mathrm{DC}_{\max }$. However, no consideration has been given as to what effect reaction rate and conversion have on the final polymer structure at short- to medium-range length scales. Ordering in amorphous materials is typically classified within two length scale regimes: short and medium. Short-range order refers to bonding between atoms at length scales between $\sim 0$ and $4 \AA{ }^{28}$ such as covalent and hydrogen bonds. Medium-range ordering describes the interconnection of constituent units within a structure $(\sim 5-20 \AA),{ }^{28}$ which for densely cross-linked dimethacrylate networks includes polymer segments and cross-linking distances. The concept that an identically composed and converted material can demonstrate different structures as a consequence of polymerization rate arises from consideration of the reaction itself. At increased rates of reactive group conversion and cross-linking, the emerging structure has less time to achieve its most energetically favorable form. In contrast, slowing the reaction rate may allow the propagating polymer chains to move relatively to find a more energetically efficient state. A difference in structural order (conformation) is therefore hypothesized to exist, and such differences may influence the mechanical properties of the material. The challenge in identifying such differences exists because the likely structural differences will occur mainly at short-medium length scales. Scattering measurements using laboratory $\mathrm{X}$-ray sources fail to provide sufficient flux to discern such differences in these materials, especially when dynamic (in situ) curing studies with short measurement times are conducted.

This novel enquiry reports the determination of the crosslinked polymer structure and potential generation of strains within common biomedical photo-activated resin matrices as a function of polymerization rate through a comprehensive series of synchrotron X-ray scattering, neutron scattering combined with isotopic substitution, and complementary FTIR spectroscopic measurements. As there is no previous enquiry in this area, the null hypothesis that no relationship between polymerization rate and structural conformation of the resultant polymer network normalized to DC was tested. 


\section{MATERIALS AND METHODS}

2.1. Preparation of Photopolymerizable Dimethacrylate Resin Blends. Two dimethacrylate monomers, bisphenol-A-glycidylmethacrylate (Bis-GMA) and triethyleneglycol-dimethacrylate (TEGDMA) (Sigma-Aldrich, Dorset, UK), were proportioned in 70/30, 60/40, 50/50, and 40/60 (Bis-GMA/TEGDMA) weight percentage (wt \%) ratios and combined to produce mixes. The viscosity of the blend increases with the proportion of Bis-GMA. ${ }^{29}$ The monomer blends were combined with a photo-initiator as either 0.2 wt \% camphorquinone (CQ) with 0.8 wt \% of its tertiary amine $\mathrm{N}, \mathrm{N}$-dimethylaminoethyl-methacrylate or $1 \mathrm{wt} \%$ of the type 1 photoinitiator, Lucirin-TPO (TPO) (Sigma-Aldrich, Dorset, UK), which does not require a co-initiator. The proportioned monomers and photo-initiators were homogenized in a glass beaker using a magnetic stirrer at $50 \pm 1{ }^{\circ} \mathrm{C}$ for $30 \mathrm{~min}$ in dark conditions. The resultant resin blends were stored in dark conditions in sealed containers at $4 \pm 1{ }^{\circ} \mathrm{C}$ prior to further use.

2.2. Preparation of Prepolymerized Partially Deuterated Dimethacrylate Resin Blends. To characterize structure at crosslinking distances, the methacrylate end groups of TEGDMA were isotopically substituted with deuterium. Deuterium has a relatively large coherent neutron-scattering cross-section ${ }^{30}$ and scatters neutrons more efficiently than its lighter counterpart. Isotopic substitution was undertaken for the TEGDMA monomer only, producing $\mathrm{d}_{10}$-TEGDMA (Figure 1). Details regarding the synthesis of $\mathrm{d}_{10}$-TEGDMA are included in the Supporting Materials and Methods.

Partially deuterated resin blends were produced in a way similar to those with a hydrocarbon methacrylate functionality described in 2.1, but with TEGDMA substituted with $\mathrm{d}_{10}$-TEGDMA. As deuterated monomers were produced in significantly smaller quantities, only $60 /$ 40 and 40/60 wt \% blends were prepared.

Resin disc-shaped specimens were fabricated by filling a polyvinylsiloxane mold (11 $\mathrm{mm}$ diameter and $1 \mathrm{~mm}$ in depth) and then covered with a microscope cover slide to minimize the formation of an oxygen inhibition layer. Specimens were photopolymerized using an EMS Swiss master light curing unit (EMS OPTIDENT, electro medical systems, Nyon Switzerland) placed normal to and in contact with the cover slide. The curing unit has a spectral output range of $390-550 \mathrm{~nm}$ to provide significant overlap with the absorption peaks of the CQ $(470 \mathrm{~nm})$ and Lucirin TPO $(381 \mathrm{~nm})$ photo-initiators. For each unique composition, the resin was either photopolymerized at relatively "high" $\left(3000 \mathrm{~mW} \mathrm{~cm}^{-2}\right.$ for $6 \mathrm{~s}$ ) or "low" ( $300 \mathrm{~mW} \mathrm{~cm}^{-2}$ for $60 \mathrm{~s}$ ) irradiances ensuring matched total energy doses or radiant exposures $\left(18 \mathrm{~J} \mathrm{~cm}^{-2}\right)$. The resultant specimens were stored in lightproof conditions in sealed containers at $4 \pm 1{ }^{\circ} \mathrm{C}$ prior to further use. Samples were purposely manufactured to be no more than $1 \mathrm{~mm}$ in thickness to reduce the effects of absorption and incoherent and inelastic scattering, which is significant in materials containing hydrogen.

2.3. Simultaneous X-ray Scattering and FT-NIR Spectroscopy Measurements. Simultaneous synchrotron radiation (SR) X-ray scattering/Fourier transform-near infrared (FT-NIR) spectroscopy measurements were performed on the I22 beamline (Diamond Light Source, Oxford, UK). An incident X-ray energy of $12 \mathrm{keV}$ was used corresponding to a wavelength of $1.033 \AA$ with a beam size of $0.32 \times$ $0.08 \mathrm{~mm}$ (horizontal $\times$ vertical). Specimens were prepared by filling a $0.9 \mathrm{~mm}$ thick stainless steel ring with a $10 \mathrm{~mm}$ internal diameter, which was sealed on both faces by $25 \mu \mathrm{m}$ thick mica (Attwater Group, Lancashire, UK). The resin-filled rings were housed within a brass block annulus (Daresbury Laboratory, Warrington, UK), and a $5 \mathrm{~mm}$ diameter light guide (Lumencor, Kent, UK) was fixed normal to and $15 \mathrm{~mm}$ distant from the center of the resin surface and connected to a multichannel light source (Lumencor Aura Light engine, Lumencor, Kent, UK) and fixed in place to illuminate only the sample that was directly in the path of the X-rays. Samples were mounted to impinge the X-ray beam at a $40^{\circ}$ incline in the positive azimuthal direction. For the TPO-initiated resins, a filter within the light engine was used to emit a peak emission spectrum at $405 \mathrm{~nm}$, while for CQ systems a wavelength of $470 \mathrm{~nm}$ was employed. The light filters and their corresponding wavelengths were chosen to achieve the greatest overlap with the maxima in the visible region of the absorption spectra of the respective photo-initiators. ${ }^{31}$ For each unique composition, samples of monomer blends $(n=3$, per composition) were photopolymerized at four different irradiances for each wavelength controlled by the power output of the light engine $(5,20,60$, and $100 \%$ power output) for a total of $300 \mathrm{~s}$. Irradiances were calculated by spectrometry measurements for each testing setup, light source, and power setting and are reported with the experimentally related scattering data. 2D X-ray scattering patterns were collected every second (1 s readout) throughout polymerization with a Pilatus (P3$2 \mathrm{M}$ ) detector providing a $q$ range of $0.1-1.6 \AA^{-1}$, where $q=4 \pi / \lambda$ sin $\theta$. Additional measurements were made using a $400 \mu$ s time resolution to confirm that a $1 \mathrm{~s}$ capture rate, used for all other scattering measurements, accurately characterized dynamic structural changes.

$\mathrm{X}$-ray measurements were acquired for the constituent monomers (Bis-GMA and TEGDMA) in isolation and containing photo-initiator to identify their contributions to the scattering signal of each blend through weighted least squares (WLS) fitting. The contribution of the $\mathrm{X}$-ray beam to the measurements was assessed by conducting observations for over $30 \mathrm{~min}$ for the uncured and polymerized samples in the absence of light. To aid data analysis, measurements were taken for direct beam, empty sample containers, mica windows, water, and a silver behenate calibration standard to allow the scattering patterns to be corrected for background and normalization effects. Transmitted beam was recorded throughout using a beamstop mounted photodiode. In addition to in situ photopolymerization measurements and control/calibration measurements, scattering patterns were obtained for control molecules that possess structures similar to those under investigation [methacrylic acid (MA), methyl methacrylate, ethylene glycol, triethylene glycol (TEG), bisphenol A, and bisphenol A dimethacrylate (all Sigma-Aldrich, Dorset, UK)]. The data were normalized and background corrected using the DAWN software package (version 2.8.0, 2017, Diamond Light Source, Oxford, UK).$^{32,33}$ Data were azimuthally integrated over $360^{\circ}$ to produce a $1 \mathrm{D}$ output and were subsequently fitted with pseudoVoigt models to obtain the peak center and the full width at half maximum (fwhm).

To confirm the observations from the $\mathrm{I} 22$ beam line, additional synchrotron X-ray scattering measurements were performed on the $\mathrm{XMaS}$ beam line (BM28) at the European Synchrotron Radiation Facility (ESRF, Grenoble, France). An incident X-ray energy of 15 $\mathrm{keV}$ was used corresponding to a wavelength $(\lambda)$ of $0.82 \AA$, with a beam size of $0.08 \times 0.5 \mathrm{~mm}$ (horizontal $\times$ vertical). A similar experimental geometry was used to that described in Section 2.3 and additional details for this experiment can be found in the Supporting Materials and Methods.

In situ real-time FT-NIR spectroscopy was incorporated into the beamline setup to obtain simultaneous (to the scattering data) measurements of reactive group conversion throughout photopolymerization to enable $\mathrm{DC}_{\max }$ and maximum reaction rate $\left(R_{\mathrm{pmax}}\right)$ to be calculated. Optical (emitting and receiving) fibers $(0.6 \mathrm{~mm}$ core diameter) (Hellma Analytics, Essex, UK) were placed either side of the sample at a $45^{\circ}$ incline relative to the sample face and connected to a Nicolet IS50 spectrometer (Thermo Scientific, Warrington, UK), Figure 2. During photopolymerization, NIR spectra were collected in transmission mode using a white light source and an InGaAs detector. Real-time NIR spectra $\left(4000-10000 \mathrm{~cm}^{-1}\right)$ were collected in transmission mode $\left(4 \mathrm{~cm}^{-1}\right.$ spectral resolution) for $300 \mathrm{~s}$ with an integration time of $0.3 \mathrm{~s}$. Reactive group conversion was determined from the aliphatic $\mathrm{C}=\mathrm{CH}_{2} \mathrm{IR}$ absorption band $\left(6164 \mathrm{~cm}^{-1}\right)$, located within the methylene functional end groups of the Bis-GMA and TEGDMA monomers. As polymerization progressed, this absorption peak decreased as $\mathrm{C}=\mathrm{C}$ bonds were converted to single $\mathrm{C}-\mathrm{C}$. Conversion of the vinyl bonds (DC) is given as a percentage of the initial reagent $\mathrm{C}=\mathrm{C}$ concentration

$$
\mathrm{DC}=\left(1-\left(\frac{=\mathrm{CH}_{2}^{\text {polymer }}}{=\mathrm{CH}_{2}^{\text {monomer }}}\right)\right) \times 100
$$


Pilatus (P3-2M) Detector

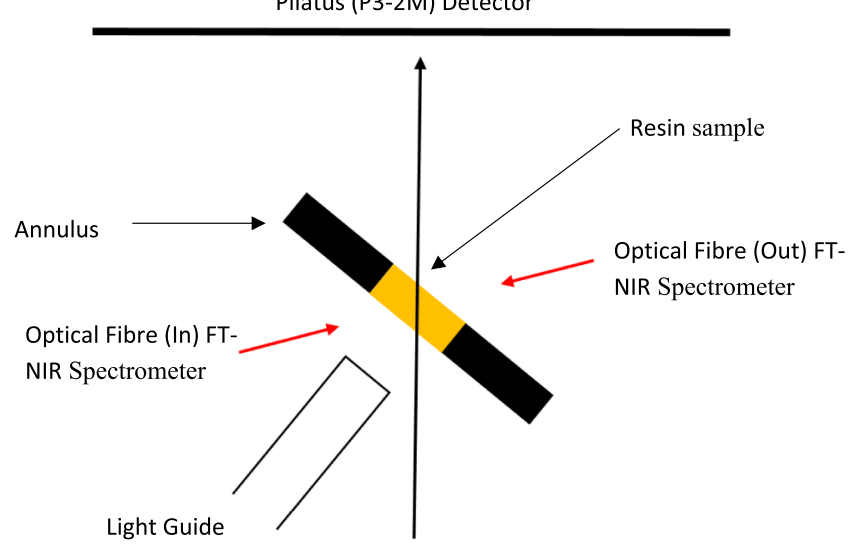

Direction of Impinging X-rays

Figure 2. Experimental setup for simultaneous synchrotron X-ray scattering and real-time FT-NIR spectroscopy measurements. The resin samples were cured for $300 \mathrm{~s}$ at varying light intensities while undertaking X-ray scattering and FT-NIR spectroscopy to quantify changes in the evolving polymer structure and the degree of reactive group conversion, respectively.

where $=\mathrm{CH}_{2}^{\text {monomer }}$ refers to the integrated peak intensity of the band prior to polymerization (in the monomer state), while $=\mathrm{CH}_{2}^{\text {polymer }}$ corresponds to the integrated peak intensity for the $i$ th measurement. The rate of polymerization was calculated as the first derivative of DC with respect to time. Additional spectral profiles were taken of the empty cell, mica windows, and the monomer to correct for background subtraction and intensity normalization. Data were baseline corrected using Omnic software (Omnic Specta software, version 8.0, Thermo Fisher Scientific, Oxford, UK).

Separate laboratory FT-NIR spectroscopy measurements, replicating the sample geometry and irradiance regimen described in Section 2.3, were performed to confirm the observations of in situ observations (see Supporting Materials and Methods).

2.4. Small-Angle Neutron Scattering. Small-angle neutron scattering (SANS) measurements were performed on the SANS2D ${ }^{34}$ instrument at the ISIS pulsed neutron source (Rutherford Appleton Laboratories, Oxford, UK). Disc-shaped partially deuterated resin samples were sealed within aluminum foil and mounted on a cadmium sample rack. SANS2D is a time of flight instrument which uses a wavelength range of $1-12 \AA$ to provide a $q$ range of $0.004-2$ $\AA^{-1}$. An $8 \mathrm{~mm}$ (diameter) beam size was used to collect SANS data in a transmission mode geometry using a $2 \mathrm{D}$ area detector (Ordela, ${ }^{3} \mathrm{He}-\mathrm{CF}_{4}$, Oak Ridge National Laboratory, Tennessee, USA). ${ }^{35}$ Acquisition times per sample were approximately $1 \mathrm{~h}$ and additional measurements were also taken on samples with a hydrocarbon methacrylate functionality to serve as controls. Further measurements were taken for the direct beam, empty sample rack, sample transmission, aluminum foil, and a vanadium calibration standard to correct for background and transmission effects and to normalize the data to absolute scattering units, respectively. Data were reduced to 1D in absolute scattering units using the SANS2D plugin for the Mantidplot ${ }^{36}$ software package (version 3.8.0, 2016, Oxford, UK).

2.5. Thermal Changes during Photopolymerization. Thermal variations associated with photopolymerization were characterized and correlated with X-ray scattering data. Temperature measurements were performed at a frequency of $10 \mathrm{~Hz}$ using type $\mathrm{K}$ thermocouples with a $0.2 \mathrm{~mm}$ tip and a Pico TC-08 data logger (Pico Technology, $\mathrm{UK})$. Ring molds equivalent to those used for X-ray scattering measurements were filled with a 60/40 wt \% Bis-GMA/TEGDMA monomer blend with either a CQ or TPO photo-initiator. The monomer blends were photopolymerized at an irradiance of $\sim 730$ $\mathrm{mW} \mathrm{cm} \mathrm{cm}^{-2}$ using a Bluephase Style 20i LED curing light (Ivoclar Vivadent, Schaan, Liechtenstein) for $180 \mathrm{~s}$. For each photo-initiator system, three measurements were conducted with the thermocouple

tip placed centrally within the resin and an additional three with the tip at the sample surface. For each thermocouple position, corresponding measurements on an empty mold, a mold containing monomer with no photo-initiator, and a mold containing the polymerized polymer were taken for $180 \mathrm{~s}$ to allow heating attributable to the curing light to also be estimated.

2.6. Simultaneous X-ray Scattering and Heating Measurements. To correlate temperature with changes in the polymer structure, synchrotron X-ray scattering experiments were performed on the I16 beamline at the Diamond Light Source (Oxford, UK). ${ }^{37}$ An incident X-ray energy of $12 \mathrm{keV}$ was used corresponding to a wavelength $(\lambda)$ of $1.033 \AA$, with a beam size of $30 \mu \mathrm{m}$ (vertical) $\times 200$ (horizontal) defined by vacuum tube slits. Measurements were carried out in air with light excluded.

A bespoke heating module was used to increase the temperature of a prepolymerized 60/40 wt \% Bis-GMA/TEGDMA resin disc specimen (10 $\mathrm{mm}$ diameter and $0.9 \mathrm{~mm}$ thickness) produced according to the previously described methods, while undertaking simultaneous X-ray scattering measurements. The heating module consisted of an aluminum plate $(25 \times 25 \mathrm{~mm}$ area $)$ with a $3 \mathrm{~mm}$ (diameter) circular aperture, to allow for the transmission of X-rays, fixed in contact perpendicular to a kinematic heating platform. The temperature of the heating platform was remotely controlled via a cryogenic temperature modulator (model 3.8, Lake Shore Cryotronics, Elliot Scientific Ltd, Hertfordshire, UK). Polymer disc specimens were held in tight contact between two aluminum plates. The temperature of test specimen was increased through heat transfer from the kinematic stage to the aluminum plates and finally to the resin disc, at a rate of $5^{\circ} \mathrm{C} \mathrm{min}-1$. Three thermocouples were placed in contact with the kinematic stage, aluminum plates, and the resin disc, respectively, to measure the temperature of each component. The heating module and housed sample were orientated with the annulus of the aluminum plate normal to the path of the impinging $\mathrm{X}$ rays.

$\mathrm{X}$-ray scattering measurements were taken at $5{ }^{\circ} \mathrm{C}$ increments, from 20 to $55{ }^{\circ} \mathrm{C}$, to characterize structural changes as a function of temperature. X-ray scattering data were collected using a $2 \mathrm{D}$ area detector (Pilatus 100K) with a $487 \times 195$ pixel format (pixel size $=$ $\left.172 \times 172 \mu \mathrm{m}^{2}\right)$ fixed on to a two theta detector arm. The detector was placed $470 \mathrm{~mm}$ behind the sample and collected scattering data at angles of $2 \theta=7,13$ and 22 to cover a $q$ range of $0.2-1.8 \AA^{-1}$, where $q$ $=4 \pi \sin \theta / \lambda$ for each $5{ }^{\circ} \mathrm{C}$ temperature increment. Scattering data were collected with a $1 \mathrm{~s}$ count time and a $2.7 \mathrm{~ms}$ readout time. Transmitted beam was also recorded at each angle and temperature increment using a photodiode to correct for changes to the sample density and thickness. To aid data analysis, measurements were taken for direct beam, empty sample containers, and a silver behenate calibration standard. Scattering patterns were background corrected and normalized to the incident monitor intensity. Data were azimuthally integrated over $360^{\circ}$ to produce a $1 \mathrm{D}$ output and were subsequently fit with a pseudo-Voigt model to determine peak positions of correlation lengths as a function of temperature.

2.7. Statistical Analysis. A one tailed paired Students $t$-test was used to determine differences in the change in correlation length $(\alpha=$ $0.05)$, where factors were photo-initiator chemistry, irradiance protocol, and relative differences in resin matrix viscosity. Statistical analysis was performed in the R programming (version 3.1.3, 2015, R Foundation).

\section{RESULTS}

3.1. Deconvolving the Contributions of Monomeric Components to the Scattering Signal. Figure 3a shows the radially averaged X-ray scattering profile for a TPOinitiated 60/40 wt \% Bis-GMA/TEGDMA resin blend in its monomer form prior to photopolymerization (solid black line) and in the polymer state at $6 \mathrm{~s}$ of $300 \mathrm{~s}$ of constant irradiation at an intensity of $450 \mathrm{~mW} \mathrm{~cm} \mathrm{~cm}^{-2}$ (dotted black line). We observed a broad scattering feature, typical in all observed resin blends, with a peak initially located at $q \approx 1.35 \pm 0.0024 \AA^{-1}$ 

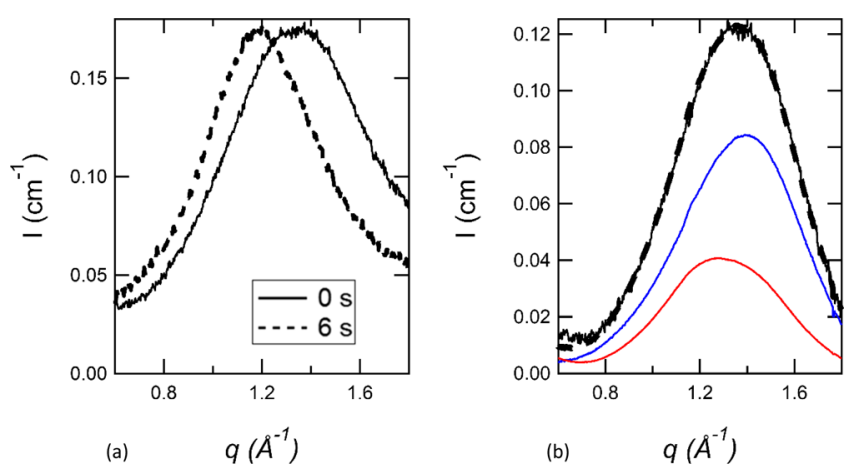

Figure 3. Representative plots showing (a) X-ray scattering of a 60/ 40 wt \% Bis-GMA/TEGDMA resin blend initiated with TPO using constant light application at an intensity of $450 \mathrm{~mW} \mathrm{~cm}^{-2}$ for $300 \mathrm{~s}$. Prior to polymerization (bold black line, at $0 \mathrm{~s}$ ), a broad scattering feature is centered at $q \approx 1.35 \AA^{-1}$, which narrows and moves to $q \approx$ $1.25 \AA^{-1}$ (broken black line, measured at $6 \mathrm{~s}$ ). (b) WLS fitting (broken black line) of scattering data from the individual Bis-GMA (red) and TEGDMA (blue) monomers to the 60/40 wt \% blend (solid black line) following baseline corrections. Bis-GMA and TEGDMA contribute 30 and $70 \%$ to the signal, respectively. Data were obtained from I22 (Diamond Light Source).

corresponding to a correlation length of $4.65 \AA \AA$ in real space, which moved to lower values of $q$, approximately $1.23 \pm$ $0.0024 \AA^{-1}(5.1 \AA)$ and incrementally narrowed throughout photopolymerization. The shift to lower $q$ is indicative of a net length increase of the correlation length, while a narrowing of the peak demonstrates an increase in the medium-range $(<10$ A) relative structural order.

Figure $3 \mathrm{~b}$ demonstrates the WLS fitting of Bis-GMA and TEGDMA X-ray scattering spectra to the signal of the 60/40 wt \% resin blend. WLS helped to identify the structural origin responsible for the peak shift with respect to individual monomer contributions to the observed signal. TEGDMA was the predominant contributor to the observed scattering peak $\left(q \approx 1.35 \AA^{-1}\right)$ and its shift, accounting for $70 \%$ of the shift, while only $30 \%$ was attributed to Bis-GMA. The contribution of each monomer to the signal varied with the composition of the resin blend.

Figure 4 illustrates the chemical structures of TEGDMAs' constituent monomers (MA and TEG). Figure 5 shows the fitting of the TEGDMA X-ray scattering signal based upon the scattering data for the constituent monomers to approximate its chemical structure. WLS fitting reveals that $\sim 68 \%$ of the TEGDMA signal was attributed to MA, representing the methacrylic functional end groups (34\% per end group) (Figure 4 ). The remaining $\sim 32 \%$ of the TEGDMA signal contribution originated from its ether backbone. Furthermore, $\sim 48 \%$ of the $60 / 40 \mathrm{wt} \%$ Bis-GMA/TEGDMA (total) signal was attributed to MA (representing the methacrylate functional end groups) originating from the TEGDMA monomer and $\sim 22 \%$ was weighted to the TEG component (representing the ether backbone) of the same monomer. Only $\sim 30 \%$ of the change in the scattering peak shift and narrowing following photopolymerization of the resin-blend was ascribed to BisGMA. For a $60 / 40$ wt \% blend, the non-aromatic segments of the Bis-GMA monomer contribute $\sim 30 \%$ to the total molecular weight of the $60 / 40$ wt $\%$ blend. This was equivalent to the contribution that Bis-GMA makes to the scattering signal for the broad feature observed in Figure 3.<smiles>C=C(C)C(=O)OCCOCCOCCOC(=O)C(=C)C</smiles><smiles>C=C(C)C(=O)OCCOCCOCCO</smiles>

Figure 4. Chemical structures of Bis-GMA and TEGDMA monomers used to formulate each blend. MA and TEG were used to simulate the chemical structure and dynamic behavior of the TEGDMA monomer during polymerization.

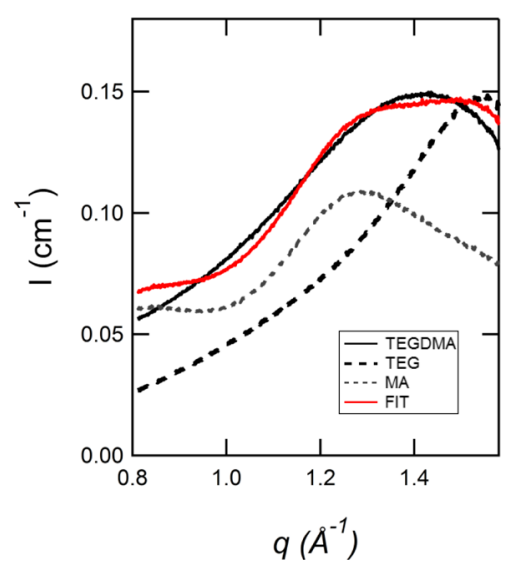

Figure 5. WLS fitting of MA and TEG X-ray scattering to that of TEGDMA. MA and TEG were used to approximate both the structure and X-ray scattering pattern of the TEGDMA monomer. Close to $70 \%$ of the shift and narrowing in scattering following polymerization was attributed to MA with the remainder ascribed to TEG. Data were obtained from I22 (Diamond Light Source).

Figure 6 shows the 1D neutron scattering spectra for TPOinitiated 60/40 wt \% (Bis-GMA/ $\mathrm{d}_{10}$-TEGDMA) resin blends, either prior to polymerization as the liquid monomer (solid black line) or after, when photopolymerized at either relatively fast $\left(3000 \mathrm{~mW} \mathrm{~cm} \mathrm{~cm}^{-2}\right.$, black dots) or slow $\left(300 \mathrm{~mW} \mathrm{~cm}{ }^{-2}\right.$, broken black line) rates. It can be seen that a broad scattering peak is initially located at $\sim 0.69 \AA^{-1}(\sim 9.1 \AA$ ), which shifts to lower $q$ and narrows in width following photopolymerization, indicative of a net increase in the correlation length and medium-range order that this scattering peak represents. Systems polymerized at relatively fast rates demonstrated a greater peak shift to lower $q \approx 0.57 \AA^{-1}(\sim 11 \AA)$ compared 


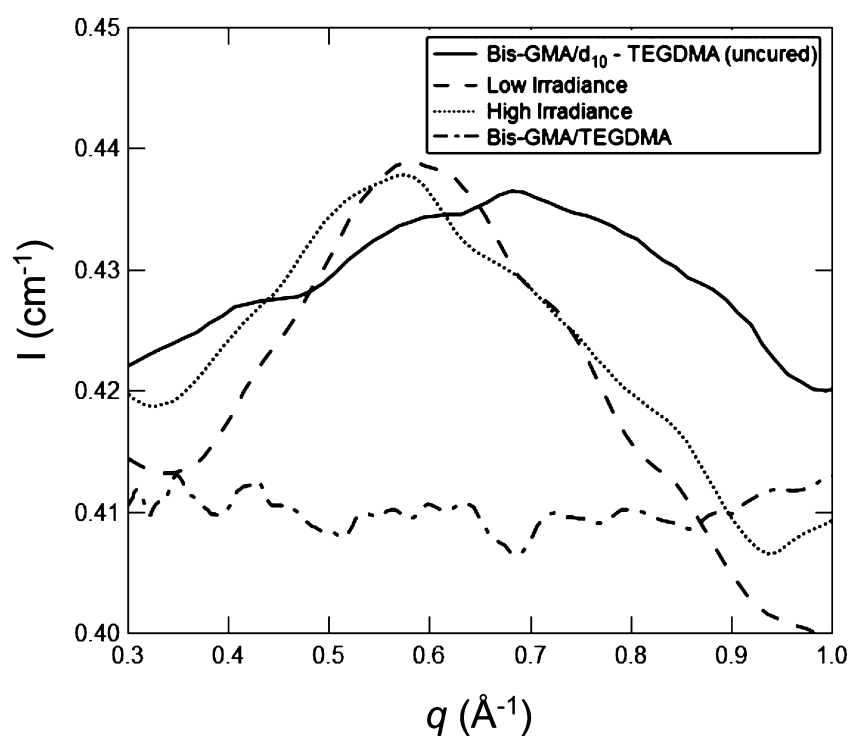

Figure 6. Representative plot of neutron scattering spectra for resins with deuterated or hydrocarbon methacrylate functionality based on a $60 / 40$ wt $\%$ blend of Bis-GMA/ $\mathrm{d}_{10}$-TEGDMA or Bis-GMA/ TEGDMA, respectively. A broad scattering peak is observed for the deuterated liquid monomer blend at $\sim 0.68 \AA^{-1}$ (-), which shifts to lower $q$ and narrows following photopolymerization. Accelerating polymerization through high light irradiance confers a greater shift to lower $q(\cdots)$ compared with slower polymerization (---). Blends with a hydrocarbon methacrylate functionality display no structural features within this $q$ range $(---)$.

with slower polymerization, where $q \approx 0.62 \AA^{-1}(\sim 10.1 \AA)$. Similarly, systems polymerized at relatively low and high irradiances displayed fwhm values of $\sim 0.13$ and $0.15 \AA^{-1}$, where a reduced peak width corresponds to greater structural order. Figure 6 also displays the neutron scattering spectra of a 60/40 wt \% (Bis-GMA/TEGDMA) resin blend (dasheddotted line) with a hydrocarbon methacrylate functionality, which displays no scattering features in the region up to $q \approx 1$ $\AA^{-1}$.

3.2. Effect of Polymerization Rate on the Evolving Polymer Structure. Figure 7 illustrates the real-time behavior of the correlation length and increase in the relative structural order for a 50/50 wt \% resin blends. The correlation length corresponds to the average bond length as taken from the peak position in Figure 3a, while the relative structural order refers to the distribution of correlation lengths calculated from the fwhm of the same scattering peak. This formulation displays typical structural changes observed in all of the resin blends for varying viscosities at relatively fast and slow polymerization rates (controlled by light intensity and photo-initiator chemistry). The peak position of the broad scattering feature observed in Figure 3a is shown in real space $(\AA)$ for each time point (every second) during light irradiation, representing an increase in the correlation length (Figure 7a,b).

Resins polymerized at faster and slower reaction rates both displayed an increase in the correlation length. For slower polymerization, typically initiated with CQ for all light intensities, this increase occurs in two stages; a relatively large and fast increase to a local maxima followed by a gradual gain with time (Figure $7 \mathrm{a}$, red line). Increasing the polymerization rate (initiating with TPO and photocuring with higher irradiance) conferred a significantly greater increase in the correlation length $(p<0.01)$ in a shorter period of time
(Figure $7 \mathrm{~b}$ ), with the magnitude of this initial extension more than double that observed at the lowest polymerization rate (Figure 7a). An additional decrease in length is also observed following initial extension for faster polymerization and is equal to approximately $5-15 \%$ of the initial extension, depending on the intensity of the activating light (see Supporting Data for additional results). A small subsequent increase was observed in the correlation length for most blends transitioning either directly from the initial extension or from this relaxation, depending on the polymerization rate. It can be seen that the final (observed) correlation length remained smaller for more slowly polymerized resins. The magnitude of the gradual extension phase is less for faster polymerization and always followed relaxation. The slow increase in the correlation length was larger when the system was polymerized with a lower light irradiance, whereas resins polymerized more rapidly show little or no increase.

Figure $7 \mathrm{c}, \mathrm{d}$ shows the increase in relative structural order, which provides insight into the distributions of chemical bond lengths and angles for fast and slower polymerization rates as a function of time. An initial increase in structural order to a local maximum occurs simultaneously with similar changes seen in the correlation length. Faster polymerization corresponded to a larger and more rapid gain in structural order. Additionally, fast polymerized systems also demonstrated a relaxation in structural order (see Supporting Data, Figure S6), where approximately $5-15 \%$ of the initial gain was lost, mirroring changes in the correlation length (see Supporting Data, Figure S5). Similarly, at slower reaction rates these systems display negligible or non-existent relaxation (see Supporting Data, Figures S3 and S4). Relative orders do not however show a postrelaxation increase as was observed in the correlation length for all polymerization rates, instead appearing to plateau (at least for the duration of the measurements). The final (measured) relative value of structural order was on average greater in resins containing a higher proportion of TEGDMA monomer (see Supporting Data, Figures S4 and S6). These less viscous systems also showed greater differences in the final relative structural order, for identical blends polymerized at different rates.

The relationship between functional end group conversion and structural changes in the polymer is outlined in Figure 7 (black traces), where the data sets are overlaid for the respective polymerization rates as a function of time. TPOinitiated systems showed more rapid periods of initiation and propagation in comparison to the CQ-based resins, reaching auto-deceleration earlier. Final conversion $\left(\mathrm{DC}_{\max }\right)$ and polymerization rate $\left(R_{\mathrm{p}}\right)$ are greater in resins polymerized using TPO initiation and/or high light intensities for each blend $(p<0.05)$. The auto-acceleration period, particularly for TPO-based systems, corresponds to the large initial increase in the correlation length and relative structural order, that is, as the polymer network grows, medium-range structural order and length scales increase. When the system has reached $98 \%$ of the total reactive group conversion, the correlation length and medium-range structural order also reached a maximum for faster polymerized systems (Figure $7 \mathrm{~b}, \mathrm{~d}$ ). However, the end of auto-acceleration lagged behind the local maxima of the correlation length and structural order for the slower (CQinitiated) resin systems (Figure $7 \mathrm{a}, \mathrm{c}$ ). Post auto-deceleration cure appears to correspond to a continued increase in the correlation length. Greater post auto-deceleration cure in slowly polymerized resins corresponded to a larger gradual 
CQ $\left(25 \mathrm{~mW} \mathrm{~cm}^{-2}\right)$
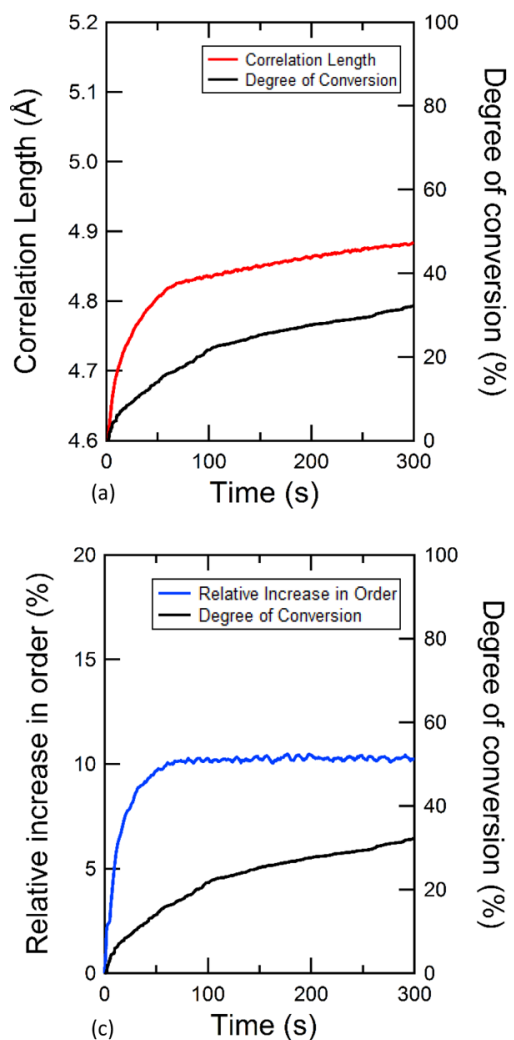

TPO $\left(730 \mathrm{~mW} \mathrm{~cm}^{-2}\right)$
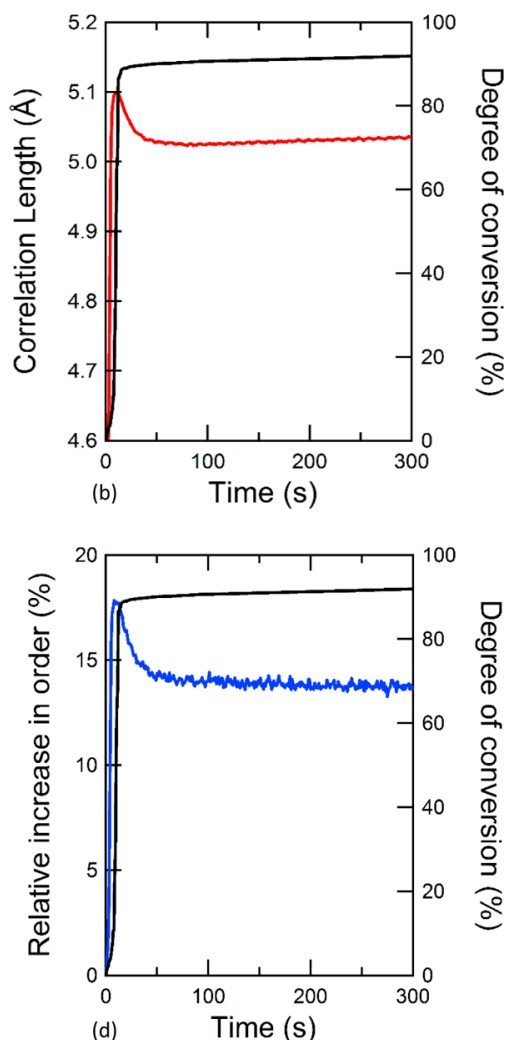

Figure 7. Representative plots of; top row: real-time evolution of the correlation length observed in 50/50 wt \% resin blends polymerized with either (a) CQ or (b) TPO photo-initiator at intensities of 25 and $730 \mathrm{~mW} \mathrm{~cm}$, respectively. All resins display an increase in the correlation length, which is larger and faster for higher polymerization rates. For particularly fast reactions, a subsequent relaxation period can be seen after the initial increase in length. Bottom row: The relative percentage increase in medium-range structural order during photopolymerization for (c) low and (d) high irradiances for the same resin blends. Changes in order are similar and occur simultaneously to those observed in the correlation length. CQ systems polymerized at irradiances of $25 \mathrm{~mW} \mathrm{~cm}^{-2}$ represent an extreme condition and it can be seen that conversion continues to rise at $300 \mathrm{~s}$. Identical systems polymerized at higher irradiances typically achieve conversion values of 55-65 \%. Data were obtained from I22 (Diamond Light Source).

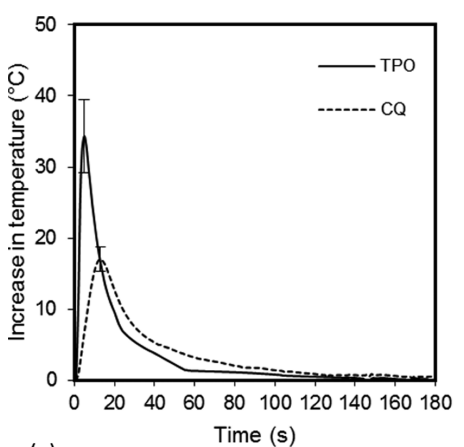

(a)

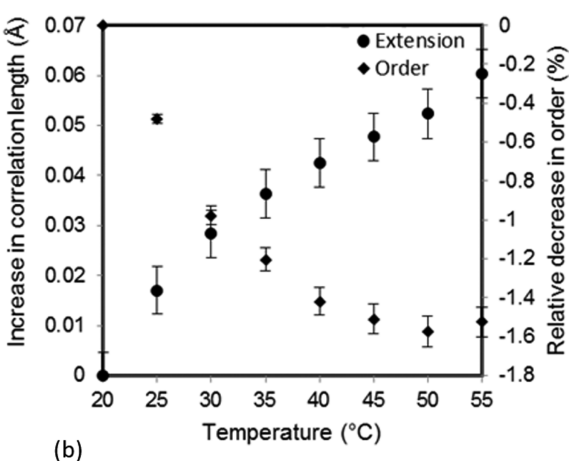

(b)

Figure 8. (a) Plot of the mean temperature rise $(n=6)$ compensated for heating from the curing light, during photocuring of $60 / 40$ wt $\%$ BisGMA/TEGDMA monomer blends containing either a CQ or TPO photo-initiator. Error bars at peak temperature rise represent one standard deviation. (b) Mean relative increase and associated error bars, obtained from the propagation of errors of peak fitting uncertainties, in the $\mathrm{X}$-ray scattering correlation length and order following controlled heating of prepolymerized $60 / 40 \mathrm{wt} \%$ Bis-GMA/TEGDMA from 20 to $55{ }^{\circ} \mathrm{C}$.

gain in the correlation length following the initial increase, while resins cured rapidly showed negligible post autodeceleration cure and changes in the correlation length. This slow continued polymerization does not appear to affect the observed relaxation period or the plateau observed in the medium-range structural order.

3.3. Effect of Temperature Variations on the Evolving Polymer Structure. Figure $8 \mathrm{a}$ illustrates the real-time temperature variations in representative 60/40 wt \% resin blends initiated with either CQ or TPO during photopolymerization. A rapid increase in the temperature of the resin blend followed by a gradual decrease is observed for both photo-initiator systems. Resins initiated with TPO demonstrate a larger temperature increase in a shorter period of time compared with CQ-based systems. Increases and relaxations in temperature during photopolymerization approximately co- 
incide with similar behavior seen in the correlation length (Figure 7a,b) and medium-range order (Figure $7 c, d$ ) for the respective photo-initiator species despite differences in sample set up. Figure $8 \mathrm{~b}$ shows the change in correlation length and medium-range order as a function of temperature for a prepolymerized $60 / 40 \mathrm{wt} \%$ resin specimen. It can be seen that heating confers an almost linear extension of the correlation length at a rate of $\sim 0.0016 \AA{ }^{\circ} \mathrm{C}^{-1}$, while order decreases by approximately $1.5 \%$.

3.4. Effect of the Polymerization Rate on the Resultant Polymer Structure. A range of polymerization rates for each resin blend were achieved by varying photoinitiator chemistry and light irradiance and were characterized by FT-NIR spectroscopy. In Figure 9, by correlating

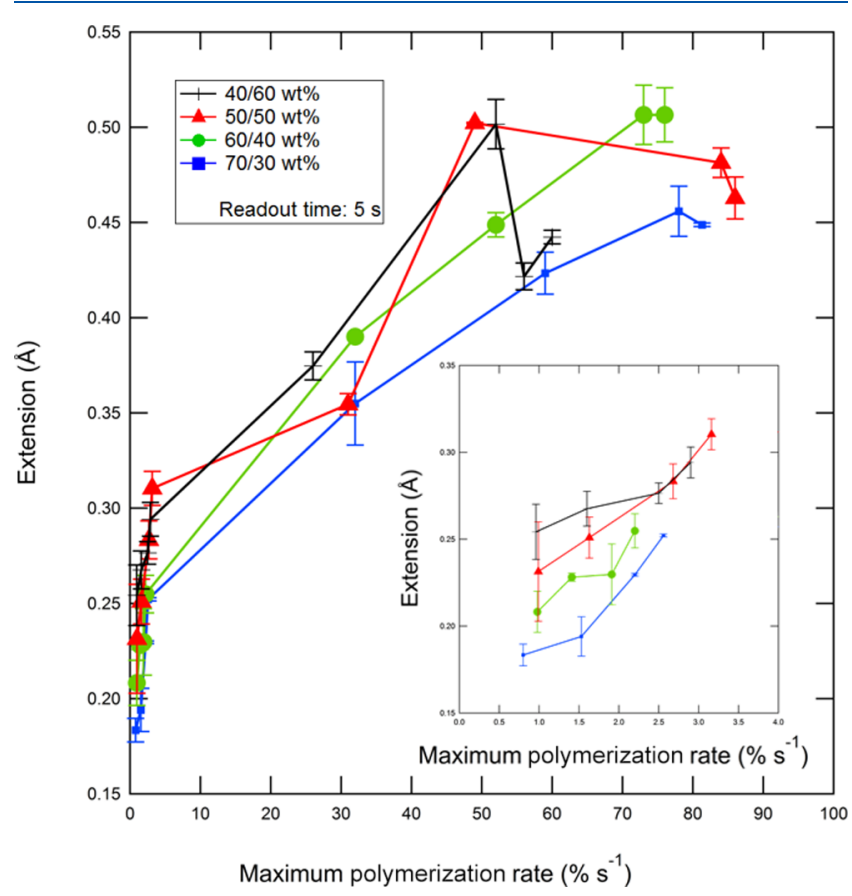

Figure 9. Maximum chain extension for each resin blend (viscosity) vs polymerization rate (irradiance). The maximum extension was calculated as the difference between the initial and greatest values of the correlation length for each blend. CQ-initiated resins are shown by the first four points and are also inset, while the other data points refer to TPO-initiated resins. Resins polymerized at higher rates display the greatest increase in the correlation length. Data were obtained from BM28 (ESRF), see Supporting Materials and Methods.

polymerization rate with changes in X-ray scattering, it was possible to relate reaction rate to (medium-range) structural evolution. As the polymerization rate increased, there was a greater increase in the correlation length (extension) and relative medium-range structural order. Figure 9 demonstrates how systems initiated with Lucirin TPO (the last four data points) display polymerization rates typically an order of magnitude greater than the CQ counterpart (the first four data points and inset in Figure 9) for similar irradiances. This conferred larger gains in the correlation length, which were approximately twice those seen in CQ systems. At lower polymerization rates $\left(<5 \% \mathrm{~s}^{-1}\right)$, the increase in the correlation length was observed to increase linearly with the polymerization rate (Figure 9); however, as the reaction rate increases, the change in correlation length begins to plateau. More fluid resins, with a greater content of TEGDMA, displayed a greater increase in the correlation length for equivalent polymerization rates. A similar relationship was observed when changes in the medium-range structural order were correlated with the polymerization rate (see Supporting Data, Figure S8), albeit that the initial increases in relative order were much greater for the more fluid systems (greater TEGDMA \%).

Direct correlations between X-ray data and the polymerization rate are complicated by the relative differences in reactive group conversion for each polymerizing system. Normalizing the increase in correlation length with respect to $\mathrm{DC}_{\max }$ resulted in a pattern of behavior similar to that observed in Figure 9. However, normalizing structural order to $\mathrm{DC}_{\max }$ and plotting as a function of polymerization rate (Figure 10) demonstrated that at polymerization rates greater than

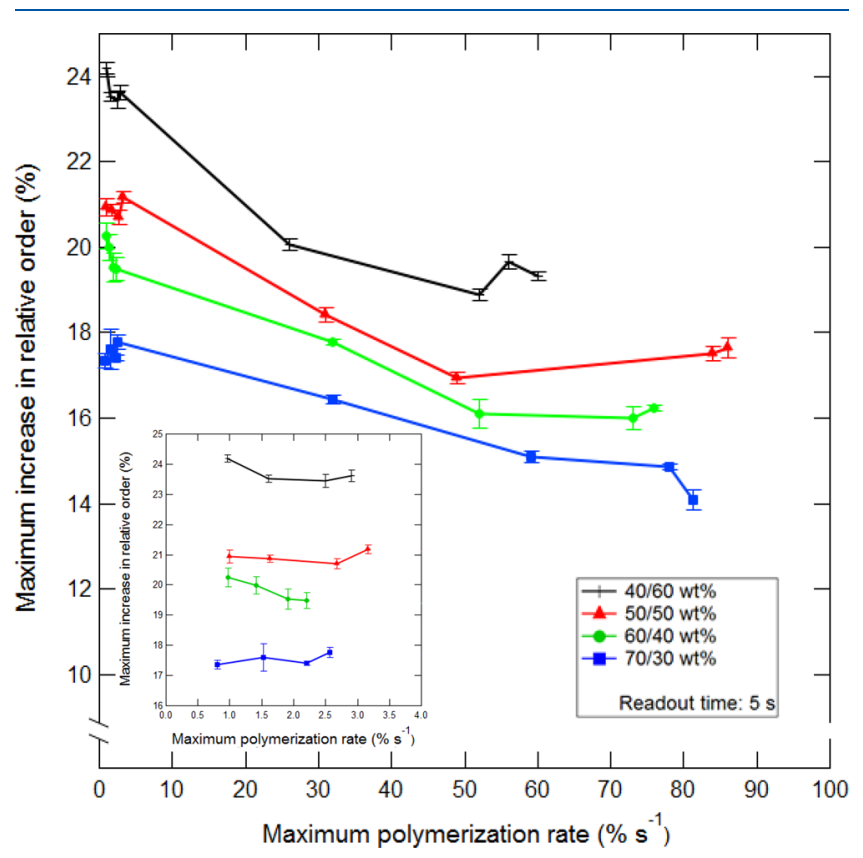

Figure 10. Normalized maximum increase in relative medium-range structural order for each resin blend (viscosity) vs polymerization rate (irradiance). Data have been normalized to the degree of reactive group conversion to determine structural order per converted $\mathrm{C}=\mathrm{C}$ bond. CQ-initiated resins are shown by the first four points (also inset), while the other data points refer to TPO-initiated resins. Systems polymerized at slower rates display the greatest increase in structural order for a given blend, while increasing viscosity decreases the medium-range order. Data were obtained from BM28 (ESRF), see Supporting Materials and Methods.

$\sim 5 \% \mathrm{~s}^{-1}$ the gain in order decreases with increasing reaction rate for all resin blends. Relative structural order remained ranked by resin "viscosity" with blends composed primarily of the Bis-GMA monomer demonstrating greater disorder at all polymerization rates.

\section{DISCUSSION}

4.1. Deconvolving the Contributions of Monomeric Components to the Scattering Signal. Photopolymerization induced an increase in the length of the principal X-ray scattering feature observed in measurements on the Bis-GMA/ TEGDMA monomer blends (Figure 3a). Previous laboratorybased studies (WAXS) have measured this broad peak feature but have failed to determine its origin or explain the subtle differences in the peak position between resin blends of varying 
viscosities, ${ }^{38}$ which have been resolved here. In this study, the correlation length increases from an initial value of $\sim 4.65 \AA$ up to $\sim 5.1 \AA$ (Figures $3 \mathrm{a}$ and $7 \mathrm{a}, \mathrm{b}$ ), depending on the resin formulation and the polymerization rate. WLS fitting of the scattering data for a 60/40 BisGMA/TEGDMA wt \% blend revealed that the majority of the length change originates from the TEGDMA monomer (Figures 4 and 5) and specifically within the methacrylate functional end groups ( $48 \%$ of the total signal). The remaining portion of this length change is ascribed to the ether backbone in the TEGDMA monomer, which accounts for the remaining $\sim 22 \%$ of the total signal.

The mechanism behind the length increase can be determined by considering that during polymerization the $\mathrm{C}=\mathrm{C}$ double bond in the methylene functional group is converted to a single covalent bond with a carbon atom from a neighboring monomer. Bond conversion confers a length increase equivalent to $0.2 \AA$ [the difference in bond length between a $\mathrm{C}=\mathrm{C}$ bond $(1.33 \AA)$ and a $\mathrm{C}-\mathrm{C}$ bond $(1.53 \AA)]^{39}$ Figures $7 \mathrm{a}$ and 9 show that for lower rates of polymerization, less than $2 \% \mathrm{~s}^{-1}$, the increases in the observed correlation length approximate the difference in length between double and single carbon-carbon bonds following polymerization. This implies that at lower reaction rates the predominant form of length change arises from bond exchange. However, at higher reaction rates, in excess of $50 \% \mathrm{~s}^{-1}$, the increase in the correlation length is more than double the bond exchange value (Figures $7 \mathrm{~b}$ and 9). This additional increase in length, which cannot be accounted for through bond exchange, is therefore ascribed to chain segment extension. $\mathrm{Fu}$ et al. $(2015)^{40}$ reported chain extension in methacrylate functionalized monomers which were photo-initiated with UV light. However, the location(s) of the chain extension were not elucidated in their study.

Chain extension along the TEGDMA backbone is further supported by neutron scattering data from partially deuterated blends (Figure 6). Figure 6 shows a broad scattering peak for a $60 / 40$ wt $\%$ (Bis-GMA/ $\mathrm{d}_{10}$-TEGDMA) liquid monomer located at approximately $0.68 \AA^{-1}(\sim 9.23 \AA)$, which shifts to lower $q$ and narrows following photopolymerization. This scattering peak is known to correspond to the correlation lengths between the deuterated portions of the functional end groups of $d_{10}$-TEGDMA, due to the absence of this scattering peak in a hydrocarbon functionality counterpart (Figure 6), and was synthesized specifically for this purpose. Therefore, this correlation length represents the approximate end-to-end vector distance of the $\mathrm{d}_{10}$-TEGDMA monomer and gives insight into the structure of TEGDMA. The increase in the correlation length is also seen in the X-ray scattering data and exceeds that which could be attributed to bond exchange. The relatively small difference in the peak shift between the high and low irradiance polymerization regimes for partially deuterated resins is attributed to fabricating the samples in advance of the neutron scattering measurements, due to the scheduling of experiments based at central facilities, which has likely permitted structural modifications to occur during a postcure period. Isotopic substitution of the functional end groups also prevents the quantification of $\mathrm{DC}_{\max }$ to normalize changes in the correlation length, which will likely reduce apparent differences in correlation length and structural order for identical systems polymerized with different irradiances. Deuterated resins polymerized at lower irradiances demonstrated greater medium-range order compared with higher irradiances similar to changes in order observed with X-ray scattering at shorter length scales. This strongly suggests that chain extension is the predominant mechanism for this length increase which occurs at sights distant from bond conversion/ cross-links and at different length scales.

The Bis-GMA monomer also displays a smaller contribution to the total X-ray scattering signal ( $30 \%)$ and subsequent $q$ shift. Any extension arising from the Bis-GMA monomer is attributed solely to the nonaromatic portion of the monomer, including the functional end groups, given the rigidity of the aromatic rings at the center of the monomer. No reports in the literature have indicated structural changes to the aromatic groups via vibrational spectroscopy methods. ${ }^{4,41}$ Microgel clusters will also inevitably form at longer length scales, although it is not possible to interpret structural properties over this range using standard $\mathrm{X}$-ray/neutron scattering approaches due to the lack of clearly definable order. Optical scattering and computational modeling ${ }^{42}$ may provide deeper insight into local density fluctuations. It must also be acknowledged that irradiance, DC, and polymerization rate will vary through the resin specimen as a function of sample thickness. ${ }^{43,44}$ Therefore, any variation in the rate of reactive group conversion and hence chain extension is averaged by both FT-NIR spectroscopy and X-ray scattering measurements, respectively. Additionally, the light source demonstrates high homogeneity and top hat factor such that lateral variation of irradiance over the face of the resin specimen is considered to be negligible.

4.2. Impact of Irradiance on the Resultant Polymer Structure. Systems polymerized relatively slowly, which were typically CQ-initiated resins and some TPO systems polymerized at low light intensities, demonstrated a gradual increase in the correlation length up to a gain of approximately $0.2 \AA$ (Figure 7a). This is also accompanied by simultaneous increases in the medium-range structural order during polymerization, both of which are synchronized with the real-time DC. Hence, polymerization of the system confers an increase in the correlation length (corresponding to segments within a monomeric unit) and medium-range structural order. In Figure $7 \mathrm{a}$, a gradual increase in the correlation length is observed representing an increasing contribution of the longer correlation lengths (arising from increasing $\mathrm{C}=\mathrm{C}$ to $\mathrm{C}-\mathrm{C}$ conversion) to the average scattering signal. It can be seen that the medium-range structural order also displays a similar initial increase which coincides with polymerization (Figure 7c) and is attributed to bond conversion. It is known that polymerization limits the range of monomer motion at the end groups, which reduces the number of possible bond conformations. ${ }^{45}$ Figure $7 \mathrm{a}$ shows that the local maxima in the correlation length correspond to plateauing of DC. Additionally, the gain in the correlation length consistently exceeds $0.2 \AA$ after a small reversal in the extension feature, which may represent a transition from chain growth-induced length changes to true chain extension. In systems polymerized at relatively slow rates, the maximum rate of conversion $\left(R_{\mathrm{pmax}}\right)$ occurs at lower reactive group conversion. ${ }^{46,47} \mathrm{~A}$ smaller fraction of monomer/ polymer segments are perturbed at this point in the reaction, the relaxation time of polymer segments $(\tau)$ is significantly less than the rate of conversion ${ }^{46}\left(R_{\mathrm{p}}\right)$, that is, $\tau \ll R_{\mathrm{p}}$, and fewer segments are stored in a higher energy conformation within the forming network. After this point in the reaction, $\tau<R_{\mathrm{p}}$, so the polymer segments have more time to extend and reorient to achieve greater structural order. Therefore, chain extension in 
relatively slowly polymerized resins seems to occur after the majority of the cross-linked network has been formed.

At faster polymerization rates (Figure $7 b, d$ ), greater increases in the correlation length and order in a shorter period of time are observed. At higher rates (TPO, high irradiance), the increase in the correlation length is more than double of that than can be ascribed to bond-exchange (associated with chain growth) and is followed by a relatively large reduction in extension length, proportional to the initial length increase. Rapid heating and cooling (Figure 8a) coincides with a large gain and relaxation in the correlation length and order, particularly for systems polymerized at faster rates through the use of higher irradiance and/or TPO photoinitiator. Thermal data indicate a strong likelihood that this temperature rise during photopolymerization in the resin contributes to the chain extension peak/relaxation seen at early time points. In particular, the peak temperature closely matches the limit of peak extension at $R_{\text {pmax }}$. However, in situ heating/X-ray scattering measurements suggest that this temperature rise may not account entirely for the extension/ relaxation behavior of the polymer segments at these early time points. Figure $8 \mathrm{~b}$ demonstrates that a greater temperature increase than seen during polymerization (Figure $8 \mathrm{a}$ ) would be required to confer the magnitude of chain extension reported in Figure 9. Furthermore, the gradual decrease in order (Figure $7 \mathrm{~d}$ ) occurs as the resin cools (Figure 8a), yet cooling would be expected to produce a relative increase in order as polymer thermal motions are reduced. This relationship is confirmed in Figure $8 \mathrm{~b}$, where heating of a $60 / 40 \mathrm{wt} \%$ resin conferred a relative decrease in order. A relaxation in order therefore supports the assertion that rapid polymerization and polymer growth generates stress within monomeric units, causing extension of segments (more so than for systems polymerized with lower reaction rates). Relaxation of these length scales may therefore represent a reorientation of bond geometry to lower the energy state of the system, that is, stress relief within the polymer structure.

Figure $7 \mathrm{~b}$ also shows that systems polymerized rapidly can demonstrate varying degrees of chain extension postrelaxation. This feature is most prominent in systems polymerized using lower light intensities, achieving lower conversion (see Supporting Data, Figure S3). In comparison, a system, which has been polymerized rapidly and has reached a high terminal conversion, displays negligible extension after the initial increase and subsequent reduction in correlation length (see Supporting Data, Figure S5). Any changes in correlation length after gelation are attributed to continued conversion, that is, bond exchange. Postcure will be associated with polymerization with less geometric freedom for the reacting monomer. This restriction of geometric freedom is likely to lead to bond and chain geometries that are longer than a comparative unconstrained state and leads to the net increase in correlation lengths seen at extended times for systems with initially lower DC.

At lower DC, more free volume is likely to be available and diffusion limits do not fully restrict the movement of radical species. This allows for continued conversion of reactive groups (Figure $7 \mathrm{a}$ ), which causes the monomer segment to incrementally extend. Resins polymerized rapidly with a greater terminal DC (>90\%) will demonstrate little final free volume. This limited mobility restricts further structural modifications such that an extended chain conformation is fixed into the polymer network. To summarize, at greater polymerization rates, $R_{\text {pmax }}$ will occur at greater values of conversion ${ }^{46,47}$ and a larger fraction of polymer segments will be perturbed. Here, $\tau$ $\ll R_{\text {pmax }}$ and the polymer segments will have insufficient time to relax $^{46,47}$ following chain extension. Consequently, higher energy (possibly under strain) extended chain segment conformations are stored within the network structure, while the cross-linked network forms and the resultant structures are unable to undergo further modifications post auto-deceleration with respect to medium-range structural order due to mobility restrictions.

4.3. Effect of the Polymerization Rate on the Resultant Polymer Structure. It can be seen from Figure 9 that as the polymerization rate is increased there is a greater increase in the correlation length (extension). At lower polymerization rates (less than $5 \% \mathrm{~s}^{-1}$ ), the relationship between extension and rate is linear; however, above this threshold, the extension begins to plateau. This plateau in extension at higher rates may represent either the maximum extension of the monomer segment, that is, toward an ideal conformation or alternatively the maximum length before solidification restricts further extension. A similar pattern is observed when changes in the medium-range structural order were correlated with the polymerization rate (see Supporting Data, Figure S8). Greater relative structural order in resins polymerized using a fast rate is likely an effect of differences in reactive group conversion. A more polymerized system will inevitably display greater structural order due to the restriction of the thermal motions and chemical bond distributions.

Direct correlations between X-ray scattering data and the polymerization rate are therefore complicated by the relative differences in reactive group conversion for each polymerizing system. DC was quantified using both real-time ex situ laboratory-based FT-NIR spectroscopy and in situ measurements performed simultaneously to the acquisition of X-ray data with differences between the two measurements considered negligible. Normalizing the increase in correlation length with respect to $\mathrm{DC}$ resulted in a pattern of observations similar to those seen in Figure 9 (see Supporting Data, Figure S7). However, Figure 10 shows that normalized structural order decreases with increasing reaction rate. This implies that driving the polymerization rate faster reduces the amount of time that the monomeric structure has to reorient and optimize its bond geometry during network growth. At slower polymerization rates, more time is allowed to achieve a lower energy (a more ordered) conformation.

The generation of macroscopic shrinkage ${ }^{48}$ associated with the reduction of free volume during the polymerization of these materials must be considered when interpreting these results. When shrinkage is constrained, the result is the generation of residual stress which will likely impact on the observed correlation lengths within the polymer system. The geometry of the test specimens used in this study was largely unconstrained with a large aspect ratio between the disc surface and the sample holder, with a readily deformable mica window. Although shrinkage has not been measured in this study, residual shrinkage strains would manifest as increased correlation lengths that may relax over time. When polymerization is accelerated through the use of TPO initiator and the highest irradiance, a relaxation feature is observed. It is conceivable that this feature is a form of stress relief mechanism, such as bond exchange or breakage. 


\section{CONCLUSIONS}

The evolving polymer structure of photo-activated dimethacrylate resins has been studied using neutron scattering, time resolved X-ray scattering, and in situ FT-NIR spectroscopy as a function of polymerization rate. Photopolymerization induces structural changes in the monomeric units with regards to chain segment extension and relative structural order, which to date is unreported for these materials. It appears that chain extension occurs predominantly in the methacrylate functional groups of Bis-GMA and TEGDMA, although extension also occurs in the ether backbone of the TEGDMA monomer. Faster polymerization rates generate greater extension in a shorter period of time, and as a consequence the developing structure has less time to orient to a lower energy (higher order) conformation. By polymerizing the system rapidly, to the point of solidification, any residual stresses generated by chain extension remain fixed into the polymer network. In comparison, slowly polymerized systems are capable of modifying the polymer structure after the majority of the network has formed, which is most likely due to a greater availability of free volume. This series of experiments within the context of the photopolymerization of biomedical resins has shown how operator-induced, environmental and compositional variables may impact polymerization. This may ultimately explain the discrepancies in the predictive modeling of the mechanical behavior of these materials used in this field to date.

\section{ASSOCIATED CONTENT}

\section{S Supporting Information}

The Supporting Information is available free of charge on the ACS Publications website at DOI: 10.1021/acs.macromol.9b00133.

Description of $\mathrm{d}_{10}$-TEGDMA synthesis; outline of additional time-resolved X-ray scattering experiment conducted at BM28 ESRF (Grenoble, France); ex-situ FTIR; real-time evolution of the correlation length and change in relative structural order observed in CQ and TPO initiated resins; maximum chain extension for each resin blend normalized to the degree of reactive group conversion, versus polymerization rate; and maximum increases in structural order for each resin blend versus polymerization rate $(\mathrm{PDF})$

\section{AUTHOR INFORMATION}

\section{Corresponding Authors}

*E-mail: oaddison@ualberta.ca. Phone: +1 7804925308 (O.A.).

*E-mail: r.a.martin@aston.ac.uk. Phone: +44 (0)121 2045111 (R.A.M.).

ORCID

Slobodan Sirovica: 0000-0002-2293-0191

Owen Addison: 0000-0002-0981-687X

Richard A. Martin: 0000-0002-6013-2334

\section{Author Contributions}

$\triangle$ Joint last authors.

\section{Author Contributions}

O.A., R.A.M., M.W.A.S., and S.S. conceived the experiment, performed the measurements, interpreted the data, and drafted the manuscript. Y.G. conducted thermal analysis. M.P. synthesized the $d_{10}$-TEGDMA. P.B.J.T., A.J.S., and W.M.P. assisted with measurements and interpreting data. All authors revised the manuscript prior to submission.

\section{Funding}

The following funding is acknowledged: Science and Technology Facilities Council (award no. ST/L502510/1) and the Royal College of Surgeons of England Small Grants Scheme (Faculty of Dental Surgery).

\section{Notes}

The authors declare no competing financial interest.

\section{ACKNOWLEDGMENTS}

The authors would like to thank Diamond Light Source for beam time (proposals SM11687-1,SM14117 and MT15319-1) and the staff of the I16 and I22 beamlines for their assistance with data collection. We thank the Science and Technology Facilities Council (STFC) for access to the SANS2D neutron beamline (proposal 1320128) at ISIS. We also acknowledge the European Synchrotron Radiation Facility for provision of synchrotron radiation facilities (proposals 28-01-1059 and 2801-1025) and the institute Laue Langevin (proposal 9-111714) and the staff of the BM28 and D16 beamlines, respectively, for their assistance with data collection.

\section{REFERENCES}

(1) Calheiros, F.; Kawano, Y.; Stansbury, J.; Braga, R. Influence of Radiant Exposure on Contraction Stress, Degree of Conversion and Mechanical Properties of Resin Composites. Dent. Mater. 2006, 22, 799-803.

(2) Stansbury, J. W. Dimethacrylate Network Formation and Polymer Property Evolution as Determined by the Selection of Monomers and Curing Conditions. Dent. Mater. 2012, 28, 13-22.

(3) Ogunyinka, A.; Palin, W. M.; Shortall, A. C.; Marquis, P. M. Photoinitiation Chemistry Affects Light Transmission and Degree of Conversion of Curing Experimental Dental Resin Composites. Dent. Mater. 2007, 23, 807-813.

(4) Stansbury, J. W.; Dickens, S. H. Determination of Double Bond Conversion in Dental Resins by near Infrared Spectroscopy. Dent. Mater. 2001, 17, 71-79.

(5) Dickens, S. H.; Stansbury, J. W.; Choi, K. M.; Floyd, C. J. E. Photopolymerization Kinetics of Methacrylate Dental Resins. Macromolecules 2003, 36, 6043-6053.

(6) Ruyter, I. E.; Øysaed, H. Composites for Use in Posterior Teeth: Composition and Conversion. J. Biomed. Mater. Res. 1987, 21, 11-23.

(7) Ferracane, J. L. Current Trends in Dental Composites. Crit. Rev. Oral Biol. Med. 1995, 6, 302-318.

(8) Lima, A. F.; de Andrade, K. M. G.; da Cruz Alves, L. E.; Soares, G. P.; Marchi, G. M.; Aguiar, F. H. B.; Peris, A. R.; Mitsui, F. H. O. Influence of Light Source and Extended Time of Curing on Microhardness and Degree of Conversion of Different Regions of a Nanofilled Composite Resin. Eur. J. Dent. 2012, 6, 153-157.

(9) Peutzfeldt, A.; Asmussen, E. The Effect of Postcuring on Quantity of Remaining Double Bonds, Mechanical Properties, and in Vitro Wear of Two Resin Composites. J. Dent. 2000, 28, 447-452.

(10) Feng, L.; Suh, B. I. Exposure Reciprocity Law in Photopolymerization of Multi-Functional Acrylates and Methacrylates. Macromol. Chem. Phys. 2007, 208, 295-306.

(11) Emami, N.; Soderholm, K.-J. M. How light irradiance and curing time affect monomer conversion in light-cured resin composites. Eur. J. Oral Sci. 2003, 111, 536-542.

(12) Halvorson, R. H.; Erickson, R. L.; Davidson, C. L. Energy Dependent Polymerization of Resin-Based Composite. Dent. Mater. 2002, 18, 463-469.

(13) Sakaguchi, R. L.; Ferracane, J. L. Effect of Light Power Density on Development of Elastic Modulus of a Model Light-Activated Composite during Polymerization. J. Esthet. Restor. Dent. 2001, 13, 121-130. 
(14) Lovell, L. G.; Lu, H.; Elliott, J. E.; Stansbury, J. W.; Bowman, C. N. The Effect of Cure Rate on the Mechanical Properties of Dental Resins. Dent. Mater. 2001, 17, 504-511.

(15) Wydra, J. W.; Cramer, N. B.; Stansbury, J. W.; Bowman, C. N. The reciprocity law concerning light dose relationships applied to BisGMA/TEGDMA photopolymers: Theoretical analysis and experimental characterization. Dent. Mater. 2014, 30, 605-612.

(16) Sideridou, I.; Tserki, V.; Papanastasiou, G. Effect of Chemical Structure on Degree of Conversion in Light-Cured DimethacrylateBased Dental Resins. Biomaterials 2002, 23, 1819-1829.

(17) Van Landuyt, K. L.; Snauwaert, J.; De Munck, J.; Peumans, M.; Yoshida, Y.; Poitevin, A.; Coutinho, E.; Suzuki, K.; Lambrechts, P.; Van Meerbeek, B. Systematic Review of the Chemical Composition of Contemporary Dental Adhesives. Biomaterials 2007, 28, 3757-3785.

(18) Venhoven, B. A. M.; de Gee, A. J.; Davidson, C. L. Light Initiation of Dental Resins: Dynamics of the Polymerization. Biomaterials 1996, 17, 2313-2318.

(19) Lovell, L. G.; Stansbury, J. W.; Syrpes, D. C.; Bowman, C. N. Effects of Composition and Reactivity on the Reaction Kinetics of Dimethacrylate/Dimethacrylate Copolymerizations. Macromolecules 1999, 32, 3913-3921.

(20) Amirouche-Korichi, A.; Mouzali, M.; Watts, D. C. Effects of Monomer Ratios and Highly Radiopaque Fillers on Degree of Conversion and Shrinkage-Strain of Dental Resin Composites. Dent. Mater. 2009, 25, 1411-1418.

(21) Chen, Y.-C.; Ferracane, J. L.; Prahl, S. A. Quantum Yield of Conversion of the Photoinitiator Camphorquinone. Dent. Mater. 2007, 23, 655-664.

(22) Nie, J.; Andrzejewska, E.; Rabek, J. F.; Lindén, L. Å.; Fouassier, J. P.; Paczkowski, J.; Scigalski, F.; Wrzyszczynski, A. Effect of peroxides and hydroperoxides on the camphorquinone-initiated photopolymerization. Macromol. Chem. Phys. 1999, 200, 1692-1701.

(23) Monroe, B. M.; Weiner, S. A.; Hammond, G. S. Mechanisms of Photochemical Reactions in Solution. LII. Photoreduction of Camphorquinone. J. Am. Chem. Soc. 1968, 90, 1913-1914.

(24) Peutzfeldt, A.; Asmussen, E. Resin Composite Properties and Energy Density of Light Cure. J. Dent. Res. 2005, 84, 659-662.

(25) Miyazaki, M.; Oshida, Y.; Keith Moore, B.; Onose, H. Effect of Light Exposure on Fracture Toughness and Flexural Strength of Light-Cured Composites. Dent. Mater. 1996, 12, 328-332.

(26) Asmussen, E.; Peutzfeldt, A. Polymerization Contraction of Resin Composite vs. Energy and Power Density of Light-cure. Eur. J. Oral Sci. 2005, 113, 417-421.

(27) Feng, L.; Carvalho, R.; Suh, B. I. Insufficient Cure under the Condition of High Irradiance and Short Irradiation Time. Dent. Mater. 2009, 25, 283-289.

(28) Simmons, C. J.; El-Bayoumi, O. H. Experimental Techniques of Glass Science; J. Am. Ceram. Soc. 1993, 205-314.

(29) Ellakwa, A.; Cho, N.; Lee, I. The Effect of Resin Matrix Composition on the Polymerization Shrinkage and Rheological Properties of Experimental Dental Composites. Dent. Mater. 2007, 23, 1229-1235.

(30) Sears, V. F. Neutron Scattering Lengths and Cross Sections. Neutron News 1992, 3, 26-37.

(31) Stahl, F.; Ashworth, S. H.; Jandt, K. D.; Mills, R. W. LightEmitting Diode (LED) Polymerisation of Dental Composites: Flexural Properties and Polymerisation Potential. Biomaterials 2000, 21, 1379-1385.

(32) Basham, M.; Filik, J.; Wharmby, M. T.; Chang, P. C. Y.; El Kassaby, B.; Gerring, M.; Aishima, J.; Levik, K.; Pulford, B. C. A.; Sikharulidze, I.; et al. Data Analysis WorkbeNch (DAWN). J. Synchrotron Radiat. 2015, 22, 853-858.

(33) Filik, J.; Ashton, A. W.; Chang, P. C. Y.; Chater, P. A.; Day, S. J.; Drakopoulos, M.; Gerring, M. W.; Hart, M. L.; Magdysyuk, O. V.; Michalik, S.; et al. Processing Two-Dimensional X-Ray Diffraction and Small-Angle Scattering Data in DAWN 2. J. Appl. Crystallogr. 2017, 50, 959-966.

(34) Heenan, R.; King, S.; Turner, D. S.; Treadgold, J. R. SANS2D at the ISIS Second Target Station. Proc. ICANS-XVII 2006, 780-785.
(35) Duxbury, D.; Khalyavin, D.; Manuel, P.; Raspino, D.; Rhodes, N.; Schooneveld, E.; Spill, E.; Spill, E.; Terry, A. Operational performance characteristics of the WISH detector array on the ISIS spallation neutron source. J. Instrum. 2014, 9, C12008.

(36) Arnold, O.; Bilheux, J. C.; Borreguero, J. M.; Buts, A.; Campbell, S. I.; Chapon, L.; Doucet, M.; Draper, N.; Ferraz Leal, R.; Gigg, M. A.; et al. Mantid-Data analysis and visualization package for neutron scattering and $\mu$ SR experiments. Nucl. Instruments Methods Phys. Res. Sect. A Accel. Spectrometers, Detect. Assoc. Equip. 2014, 764, $156-166$.

(37) Collins, S. P.; Bombardi, A.; Marshall, A. R.; Williams, J. H.; Barlow, G.; Day, A. G.; Pearson, M. R.; Woolliscroft, R. J.; Walton, R. D.; Beutier, G.; et al. Diamond Beamline I16 (Materials \& Magnetism). AIP Conf. Proc. 2010, 1234, 303-306.

(38) Fong, H.; Dickens, S.; Flaim, G. Evaluation of Dental Restorative Composites Containing Polyhedral Oligomeric Silsesquioxane Methacrylate. Dent. Mater. 2005, 21, 520-529.

(39) Cottrell, T. L. The Strengths of Chemical Bonds; Butterworth: London, 1961.

(40) Fu, Q.; McKenzie, T. G.; Tan, S.; Nam, E.; Qiao, G. G. Tertiary Amine Catalyzed Photo-Induced Controlled Radical Polymerization of Methacrylates. Polym. Chem. 2015, 6, 5362-5368.

(41) Rueggeberg, F. A.; Hashinger, D. T.; Fairhurst, C. W. Calibration of FTIR Conversion Analysis of Contemporary Dental Resin Composites. Dent. Mater. 1990, 6, 241-249.

(42) Sarkar, S.; Baker, P. J.; Chan, E. P.; Lin-Gibson, S.; Chiang, M. Y. M. Quantifying the Sensitivity of the Network Structure and Properties from Simultaneous Measurements during Photopolymerization. Soft Matter 2017, 13, 3975-3983.

(43) Chen, Y.-C.; Ferracane, J. L.; Prahl, S. A. A Pilot Study of a Simple Photon Migration Model for Predicting Depth of Cure in Dental Composite. Dent. Mater. 2005, 21, 1075-1086.

(44) Leprince, J. G.; Leveque, P.; Nysten, B.; Gallez, B.; Devaux, J.; Leloup, G. New insight into the "depth of cure" of dimethacrylatebased dental composites. Dent. Mater. 2012, 28, 512-520.

(45) Darvell, B. W. Acrylic. In Materials Science for Dentistry, 9th ed.; Darvell, B. W., Ed.; Woodhead Publishing Series in Biomaterials; Woodhead Publishing, 2009; Chapter 5, pp 108-127.

(46) Anseth, K. S.; Kline, L. M.; Walker, T. A.; Anderson, K. J.; Bowman, C. N. Reaction Kinetics and Volume Relaxation during Polymerizations of Multiethylene Glycol Dimethacrylates. Macromolecules 1995, 28, 2491-2499.

(47) Cook, W. D. Photopolymerization Kinetics of Oligo(Ethylene Oxide) and Oligo(Methylene) Oxide Dimethacrylates. J. Polym. Sci. Part A Polym. Chem. 1993, 31, 1053-1067.

(48) Palin, W. M.; Hadis, M. A.; Leprince, J. G.; Leloup, G.; Boland, L.; Fleming, G. J. P.; Krastl, G.; Watts, D. C. Reduced Polymerization Stress of MAPO-Containing Resin Composites with Increased Curing Speed, Degree of Conversion and Mechanical Properties. Dent. Mater. 2014, 30, 507-516. 